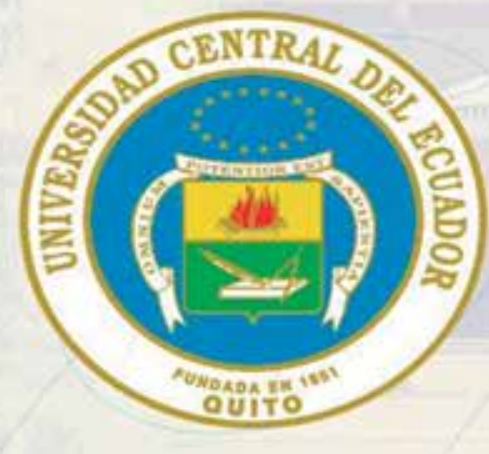

\title{
Resultados de implementación de la plataforma educativa virtual Universidad Central del Ecuador
}

\section{Identification of relevant criteria for multicriteria decision making with application of the ahp model and the Saaty scale}

\section{María José Andrade}

Licenciada en Ciencias de la Educación, mención Informática. DTIC

mjandrade@uce.edu.ec
Jorge Daniel Ortiz Herrera

Doctor en Ciencias de la Educación. Magíster en Docencia Matemática.

dortiz@uce.edu.ec

\section{Susana Cadena Vela}

Ingeniera en Sistemas e Informática.

Magíster en Educación

scadena@uce.edu.ec

\section{Resumen}

La universidad ecuatoriana principalmente ha formado profesionales, la mayoría de cursos y carreras se realizan con prácticas habituales y existe poca innovación en el uso de nuevas herramientas que favorezcan el aprendizaje, en el último lustro existieron cerca de ochenta carreras y cada una de ellas con un promedio de sesenta asignaturas. Lo común se enfoca en la transmisión de conocimiento quedando pendiente en conseguir aprendizajes, con didácticas más activas y participativas. En la búsqueda de innovar la docencia para mejorar el aprendizaje se propone la inserción de las nuevas tecnologías de información y comunicación en el quehacer universitario, realizaron la implementación de una Plataforma Virtual, cuyo principal fin es apoyar en el proceso de enseñanza-aprendizaje y cuyo producto visible es el manejo del portafolio académico para docentes y estudiantes. La implementación propuesta de la Plataforma, se realiza con un efecto permeable según los niveles de interés de la docencia, se considera como un complemento a las actividades de enseñanza y desde luego no sustituye la participación presencial del docente, más aún en que la conducción de la enseñanza radica con fuerte exclusividad en el profesorado. El primer paso para efectivizar la implementación es determinar que plataforma educativa virtual se utiliza, aquello se realiza considerando el tipo de software (Open Source), usabilidad, popularidad, soporte, código de programación, adaptabilidad y capitalización. Se han implantado aplicaciones incluyendo el concepto de e-portafolio. Las actividades y recursos (archivo, creación de cursos o módulos, tareas, etiquetas, cuestionario, foro, glosario, URL, entre otras) con las que cuenta la plataforma son variadas y permiten ser un verdadero complemento de aprendizaje para la docencia. Finalmente es importante describir los resultados tanto en la etapa de pilotaje como en la definitiva y caracterizar a través de los docentes y estudiantes participantes como parte del universo y la cantidad de cursos que se implementen.

Palabras clave: plataforma educativa virtual; proceso enseñanza aprendizaje; portafolio académico

\section{Abstract}

The Ecuadorian university has mainly trained professionals, most courses and careers are performed with usual practices and there is little innovation in the use of new tools that favor learning, in the last five years there were about eighty careers and each one with an average of sixty subjects. The common is focused on the knowledge transmission pending the achievement of learning through more active and participative didactics. In the searching to innovate tea- 
ching in order to improve learning, it is proposed the insertion of new information and communication technologies in the university work, that is done in the implementation of a Virtual Platform, with the main purpose to support in the teaching-learning process with a visible product which is the management of an academic portfolio for teachers and students. The proposed implementation of the Platform is carried out with a permeable effect according to the levels of the teaching interests, it is considered as a complement to the teaching activities and certainly it does not pretend to replace the presence of the teacher in the classroom, even more so that the teaching is rooted with a strong exclusivity in the teaching staff. The first step to make implementation effective is to determine what virtual educational platform is used, which is done considering the type of software (Open Source), usage, popularity, support, programming code, adaptability and capitalization. Applications have been implemented including in the concept of e-portfolio. The activities (file, creation of courses or modules, tasks, labels, questionnaire, forum, glossary, URL among others) that the platform counts on are varied and allow to be a true learning complement for the teaching. Finally, it is important to describe the results in both the pilot and final stages and characterize through the participating teachers and students as part of the universe and the number of courses that are implemented.

Keywords: virtual platform; teaching-learning process; academic portfolio

\section{Introducción}

Los cambios ocurridos en las últimos cien años han sido vertiginosos en relación a centurias anteriores dando paso a la creación de nuevo escenarios en donde el ser humano tiene un impacto digital, pues en toda actividad humana y social está presente la tecnología, conceptos como el ciudadano digital plantean nuevos escenarios de interacción incluso de dependencia de las tecnologías de información y comunicación. Es entonces que la educación deberá generar el espacio de aprendizaje de las tecnologías para una interacción integral, aquello provocará el aprovechamiento de las mismas.

Hay prácticas sociales masivas y personalizadas en red, constitutivas por un espacio público de nuevo tipo, pero no existe claridad en los derechos para los usuarios, aunque existen avances en muchos países que formulan políticas inclusivas y debaten el reconocimiento de esos derechos que serían fundantes para la ciudadanía digital, así como su articulación con los derechos humanos y su proyección a los entornos digitales. (Sistema de Información de Tendencias Educativas en América Latina, 2015)

"La educación que se yergue como el medio, el recurso humano civilizatorio por excelencia, fundamental para nuestro bienestar, si no se reinventa en los términos que la lógica de la digitalidad obliga, sufrirá, como sufre ahora, las profundas limitaciones de un mundo que transcurre y transpira a ritmos y velocidades diferentes" (Chanona Burguete, 2017), considerando estos conceptos la universidad deberá buscar espacios para convivir con la tecnología cumpliendo el rol de agente de cambio considerando que los "nuevos profesionales han de tener una mayor formación cultural y tecnológica además de ser más versátiles y polivalentes". (Jódar Marín, 2010)

El proceso de enseñanza-aprendizaje en carreras y programas, se desarrollaron con la réplica de modelos practicados durante varias generaciones, pues- to que su estilo de enseñanza reprodujo lo por ellos vivido en su estilo de aprendizaje, por tanto, la innovación de la enseñanza usaba de forma marginal a otro tipo de herramientas entre ellos la tecnología, de manera singular las plataformas virtuales fueron utilizadas de forma aislada y en dependencia de la iniciativa individual de los docentes.

En estas circunstancias el impulso de las plataformas tecnológicas encuentra un campo por descubrir dentro del espacio de formación superior, ello conlleva a establecer y diseñar estrategias de implementación una plataforma educativa que responda a estos nuevos escenarios de la sociedad, en donde la universidad no podría quedar aislada.

Es importante considerar el cambio de necesidad en que instituciones de Educación Superior vayan hacia la globalización e internacionalización del conocimiento (Instituto Internacional de la UNESCO para la Educación Superior en América Latina, para la Educación Superior en América Latina), por tanto contar con espacio de interacción digital es un requerimiento ineludible en los procesos de formación profesional.

Los ambientes virtuales en el contexto de la educación son espacios de interacción entre docentes y estudiantes, espacios donde se facilita la comunicación, la didáctica y la pedagógica, para intercambiar recursos didácticos textuales, multimediales e interactivos. Este espacio está disponible en todo momento para estudiantes y docentes, creando así un espacio de intercambio de información y actividades de aprendizaje, estos ambientes llamados virtuales por estar mediados por la tecnología son un apoyo tanto para la educación presencial, distancia (ahora virtual) y semipresencial. (Ardila Rodríguez, 2012).

La Universidad Central del Ecuador (UCE) es una institución que forma parte del sistema de educación superior y se encuentra facultada para otorgar títulos y grados académicos de tercero y cuarto nivel (Se- 
cretaría Nacional de Ciencia y Tecnología, 2017), la población atendida es de alrededor de los 40.000 estudiantes en 71 carreras habilitadas para titulación y 121 programas de posgrado, cuenta con 2300 docentes y 1600 servidores administrativos y de servicio.

La UCE se encuentra con una problemática generalizada en su comunidad educativa, la que se resume en el aislado uso de plataformas educativas, con ello no se favorece el desarrollo de planes de mejora en pro de mejorar la calidad educativa.

Es significativo posicionar que luego del informe que presenta el CEAACES, en la evaluación institucional 2012, se establece la obligación de construir y aplicar un "Plan Estratégico", que permita cumplir todos los requerimientos establecidos para la acreditación de la Institución, entre los que observa a la Universidad en que no dispone de una Plataforma Virtual a nivel Universitario.

A pesar de los esfuerzos aislados de las distintas Facultades y dependencias por implementar algunas herramientas no se logró consolidarlas, afectando a la mejora de la calidad de la educación.

En el trienio 2012-2014 desde la iniciativa de tecnología de información y comunicación se promocionó la plataforma Sakai, la cual se adscribió al Sistema Académico Universitario (SAU); se trató de apoyar con el Instituto Universitario de Capacitación Pedagógica (IUCP). Los resultados fueron limitados y una aproximación a explicar aquello referencia a no ligar a un plan de implementación y sostenimiento; así como no haberse generado desde las áreas de docencia o incluso investigación.

En el año 2015 la UCE inicia como proyecto la implementación de la "Plataforma Educativa Virtual", plataforma basada en Moodle, software de distribución gratuita y uno de las más utilizados en el mundo, se orienta como elemento de apoyo a los procesos de enseñanza-aprendizaje, se plantea como un elemento optativo y se inscribe la utilización del portafolio académico para el docente y estudiante, para ello se instala aplicaciones adicionales, estableciendo las siguientes premisas:

- Docencia en la modalidad presencial para apoyo a las actividades que se desarrollan en clase presencial, fomentando el aprendizaje colaborativo, manejo de portafolio académico para el docente y estudiante.

- Docencia en la modalidad a Distancia, semipresencial o virtual como herramienta fundamental para el desarrollo del proceso de enseñanza - aprendizaje, manejo de portafolio académico para el docente y estudiante.
Formación docente a cargo del IUCP, en el cual el docente podrá participar en cursos para mejorar sus competencias profesionales y de docencia.

\section{Objetivo general}

Con estos antecedentes, el objetivo de este trabajo se encuentra en "Determinar la Plataforma Educativa Virtual a utilizar, los resultados obtenidos en la implementación y la descripción del desarrollo de la actividad del docente y el estudiante".

\section{Objetivos Específicos}

- Para operativizar al objetivo general se han definido los siguientes objetivos específicos:

- Determinar la herramienta tecnológica que permita responder a las necesidades institucionales.

- Analizar las herramientas de la plataforma que son más utilizados por los docentes.

- Analizar los miembros de la comunidad universitaria que utilizan la plataforma educativa.

\section{Metodología}

La investigación acción es una metodología que apunta a la producción de un conocimiento propositivo y transformador, mediante un proceso de debate, reflexión y construcción colectiva de saberes entre los diferentes actores de un territorio con el fin de lograr la transformación social. (Montero López \& Licona Alcalan, 2016) (Loaiza \& Arévalo, 2010). Esta metodología propone las siguientes etapas: Plan de Acción, Ejecutar la Acción, Observar la Acción, Realizar las Reflexiones (Latorre, 2003) y volver a empezar con un nuevo plan de acción, por tanto este trabajo se ajusta a esta metodología con el propósito de implementar una plataforma virtual en la Universidad Central del Ecuador.

Las dos primeras etapas se describen en la metodología, y las dos siguientes en los resultados.

\section{Plan de acción}

La primera tarea y fundamental es la selección de la plataforma virtual, para la selección del Sistema manejador de Aprendizaje (LMS Learning Management System) se realiza un proyecto con la empresa PYXERA GLOBAL e IBM Corporate Service Corps, el mismo que comprende un Estudio de Factibilidad de una Plataforma Educativa Virtual para la Institución, como primera fase se realiza un análisis de tres plataformas posiblemente viables para la implementación priorizando sus fortalezas y debilidades y enfocándose a que las herramientas soporten ciertas características institucionales. 
Entre los aspectos técnicos a considerar se encuentran: el tipo de software, usabilidad, popularidad, soporte, código de programación, flexibilidad y capitalización.

Por otro lado dentro del análisis también cuenta las dimensiones de la demanda, aquello cuantificado en el número de carreras o programas, estudiantes, docentes; puesto que el rango superior está acotado en que todas las asignaturas o módulos tengan su apoyo en la Plataforma.

\section{Ejecutar acción}

Debido al volumen de usuarios se aplica la estrategia metodológica integral que facilita la difusión y el uso de las TIC en el proceso de enseñanza aprendizaje la misma se organiza de la siguiente manera:

Implementación del producto en modo pilotaje.- La implementación por fases, también llamada pilotaje de la Plataforma Virtual de Educación, es necesaria para probar el hardware y software, en una pequeña y controlada escala, por un período de tiempo que pruebe las fortalezas y debilidades del sistema. Los resultados del pilotaje serán utilizados como elementos de mejora para ser implementados en base a toda la Universidad.

- Implementación por fases.- luego del pilotaje se dimensionan los recursos tecnológicos para el despliegue total en la institución; se inicia con la fase de capacitaciones a las facultades y a los administradores de facultad que se creación para la plataforma, con la implementación de los siguientes recursos: 1) Cursos MOOC para uso de la plataforma virtual, disponible para docentes y estudiantes. 2) Módulo para solicitud de creación de curso, los cuales son verificados con el Sistema Académico Universitario para crear correctamente el nombre, asignación de estudiantes y docentes.

3) Creaciones de estructuras por dominios de trabajo es decir grado, posgrado, formación docente. 4) Políticas de uso de la plataforma educativa virtual.

\section{Resultados y discusión}

\section{Análisis y selección de plataforma:}

Se realizó el análisis de tres plataformas, OpenSWAD, Sakai y Moodle; obteniendo los siguientes resultados:

- OpenSWAD; el nombre es la abreviatura de "shared workspace at a distance" (espacio de trabajo compartido a distancia). Producto de la Universidad de Granada. Actualmente es utilizado por la Universidad Nacional de Asunción, además de la Universidad de Granada.

Fortalezas: fue desarrollado principalmente por los hispanohablantes, y esto puede ser evidente en algunos de los términos o la gramática en las páginas traducidas al inglés.

Debilidades: código base escrito en lenguaje "C", la capacidad de modificación necesita de programadores experimentados. No hay mucha ayuda fuera de línea y la popularidad es muy baja.

- Sakai; cuenta con el soporte de una fundación educativa, que supervisa el desarrollo estratégico de software, su arquitectura funciona con conjuntos de aplicaciones orientadas al servicio.

Fortalezas: dispone de una gran cantidad de foros de discusión y colaboración, que facilitan el aprendizaje; adecuado para las instituciones que requieren personalización, informes y análisis.

Debilidades: puesto que la base de código es de Java, la instalación y el costo de mantenimiento es alto; no es muy popular.

- Moodle; "Open Source", una de las plataformas virtuales de educación más utilizadas en el mundo; se apoya en una comunidad masiva y activa con numerosos "plugins" y opciones para personalizar los requerimientos de los usuarios

Fortalezas: se beneficia de una gran cantidad de documentación en línea, para solventar problemas técnicos o de usuario final; favorece el auto-aprendizaje, ya que dispone de manuales y tutoriales de libre acceso; es ajustable para instituciones que requiere una implementación rápida y confiable.

Debilidades: depende en gran medida del desarrollo de complementos de terceros cuando se requiere incorporar funcionalidades que afecten el núcleo del producto.

\section{Atributos generales de las herramientas}

En el análisis de las plataformas y los elementos que permitan determinar cuál de ellas deba escogerse, exigió el analizar los atributos que permitan al usuario tener un ambiente más amistoso y flexible, el análisis es visible en la siguiente tabla: 
Tabla 1: Atributos generales de las plataformas Fuente: Consultoría IBM-PyxeraGLobal-Universidad Central del

\section{Ecuador}

\begin{tabular}{|l|l|l|l|}
\hline Descripción & Moodle & Sakai & OpenSWAD \\
\hline Open Source & $\mathrm{Si}$ & $\mathrm{Si}$ & $\mathrm{Si}$ \\
\hline Usabilidad & Alto & Alto & Alto \\
\hline Popularidad & Alto ${ }^{*}$ & Medio & Medio \\
\hline $\begin{array}{l}\text { Soporte extensivo } \\
\text { para el español }\end{array}$ & $\mathrm{Si}$ & $\mathrm{Si}$ & $\mathrm{Si}$ \\
\hline $\begin{array}{l}\text { Código de progra- } \\
\text { mación }\end{array}$ & $\begin{array}{l}\text { PHP, MySQL } \\
\text { and Apache }\end{array}$ & Java & $\mathrm{C}$ \\
\hline Adaptabilidad & Alto ${ }^{* *}$ & Bajo & Bajo \\
\hline Capitalización & Alto ${ }^{* * *}$ & Medio & Bajo \\
\hline
\end{tabular}

* Encuestas y artículos mencionan la facilidad de uso de Moodle, se ratifica por entrevistas a docentes de la UCE.

** La versión instalada se adapta a las necesidades de la Institución.

*** La oportunidad de la UCE para ser un centro técnico para dar soporte a la versión instalada.

Genera capacidades en los estudiantes para: aprender, programar y administrar los diversos componentes; favoreciendo la innovación.

Google Trends en su análisis de popularidad y tendencia de diferentes plataformas, realiza un estudio de interés desde el año 2004 hasta la actualidad, es significativa la presencia de moodle como una plataforma, su interés es sostenido en el tiempo y supera a otras en el promedio de uso. La plataforma blackboard, bajo licencia, tiene en menor escala un nivel de aceptación significativo.

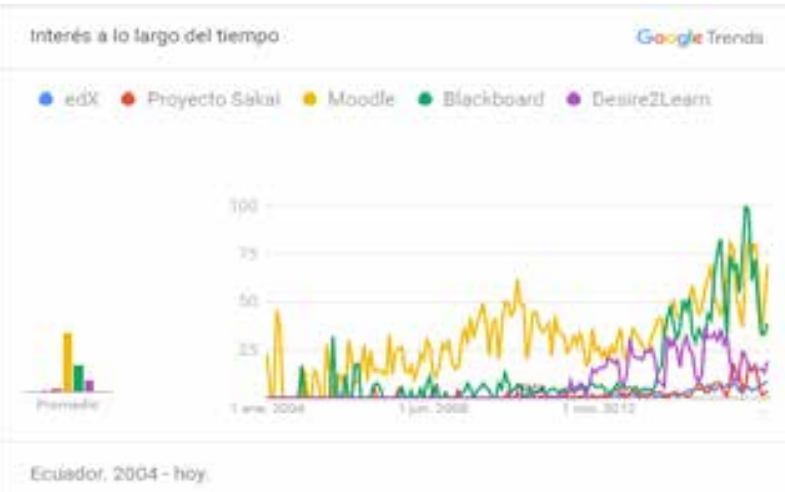

Figura 1: Estadísticas de uso de Plataformas

Fuente: Google Trends

En base al análisis y resultados precedentes la decisión se decanta por implementar la Plataforma Educativa Virtual el LMS Moodle, plataforma que cumple con las características requeridas para la Institución.
Para que la implementación de la plataforma tenga sostenibilidad y sea permeable con los actores educativos, se propone que previo a su uso se provea de soporte, aquello con la participación de la dirección de tecnologías de información y comunicación (DTIC) y de IUCP; pero el servicio debe soportarse en una política universitaria con actores del área académica.

\section{Resultado de la etapa de pilotaje}

Se motivó a grupos focales por facultades para que implementen la plataforma, los grupos debían mostrar interés para recibir el soporte técnico necesario.

Se establecen roles de acceso y se define de acuerdo a lo siguiente: Docentes: tendrán acceso a editar el curso, Estudiantes: podrán acceder a los cursos, Administrador General de la plataforma que se encuentra en la DTIC y los Administradores por cada una de las unidades académicas, entendiéndose una unidad académica una facultad, una dirección o nivelación.

Para el proceso de pilotaje se realizaron las siguientes actividades:

- Sensibilizar a los actores para que se encuentren comprometidos con la utilización de la Plataforma Educativa Virtual.

- Socialización con los docentes las políticas para la utilización de la Plataforma Educativa Virtual.

- Informar sobre las herramientas disponibles para el apoyo del proceso enseñanza-aprendizaje, los que permiten intercambiar información, generar actividades sincrónicas y asincrónicas.

- Orientarala docenciasobrelaconfiguración delaula virtual, la misma que incluye un espacio informativo, un espacio académico y un espacio de cierre; ahí se debe plantear una encuesta de satisfacción del aula virtual, la cual permita retroalimentar por parte de los participantes, el uso que se le ha dado al Aula Virtual y qué recomendaciones se pueden realizar para continuar mejorando su presentación y organización.

- Se prepara al equipo técnico de tecnología para que sean aliados en el soporte de los usuarios, de manera especial a los docentes.

- Se formula una política universitaria que se apoye desde el sector de docencia (Vicerrectorado Académico y de Investigación y Dirección General Académica). 
Luego de la configuración inicial se establecieron los requerimientos óptimos para el funcionamiento de la plataforma, estos fueron:

Tabla 2: Configuraciones óptimas para la plataforma

\begin{tabular}{|c|c|c|c|c|}
\hline $\begin{array}{l}\text { Tipo de } \\
\text { servidor y } \\
\text { S is te m a } \\
\text { Operativo }\end{array}$ & CPU & $\begin{array}{l}\text { Me- } \\
\text { Mo- } \\
\text { ria y Red }\end{array}$ & $\begin{array}{l}\text { Capaci- } \\
\text { dad de Disco }\end{array}$ & Middle- ware \\
\hline $\begin{array}{l}\text { Servidor de } \\
\text { directorio } \\
\text { Activo } \\
\text { O S : } \\
\text { Windows }\end{array}$ & 2 & $\begin{array}{l}8 \text { GB \& } 1 \\
\text { Gbit }\end{array}$ & & No aplica \\
\hline $\begin{array}{l}\text { Servidor } \\
\text { Web } \\
\text { OS: Ubuntu }\end{array}$ & $2 \mathrm{vCPU}$ & $\begin{array}{l}\text { 4GB \& } 1 \\
\text { Gbit }\end{array}$ & $\begin{array}{l}300 \text { GB SCSI con } \\
\text { bajo tiempo de } \\
\text { búsqueda }\end{array}$ & $\begin{array}{l}\text { Apacahe } \\
\text { PHP with } \\
\text { eAccellerator }\end{array}$ \\
\hline $\begin{array}{l}\text { Servidor } \\
\text { DB } \\
\text { OS: Ubuntu }\end{array}$ & $4 \mathrm{vCPU}$ & $\begin{array}{l}8 \mathrm{~GB} \& \\
1 \mathrm{Gbit}\end{array}$ & $\begin{array}{l}600 \text { GB SCSI } \\
\text { con bajo tiempo } \\
\text { de búsqueda }\end{array}$ & MySQL \\
\hline $\begin{array}{l}\text { Servidor de } \\
\text { Prueba } \\
\text { OS: Ubuntu }\end{array}$ & $2 \mathrm{vCPU}$ & $\begin{array}{l}2 \mathrm{~GB} \& 1 \\
\text { Gbit }\end{array}$ & $\begin{array}{l}100 \text { GB SCSI con } \\
\text { bajo tiempo de } \\
\text { búsqueda }\end{array}$ & $\begin{array}{l}\text { Apacahe } \\
\text { PHP con } \\
\text { eAccellerator } \\
\text { MySQL }\end{array}$ \\
\hline $\begin{array}{l}\text { Servidor de } \\
\text { Archivo } \\
\text { OS: Ubuntu }\end{array}$ & $4 \mathrm{vCPU}$ & $\begin{array}{l}6 \text { GB \& } \\
1 \text { Gbit }\end{array}$ & $\begin{array}{l}1 \text { TB SCSI con } \\
\text { bajo tiempo de } \\
\text { búsqueda }\end{array}$ & \\
\hline
\end{tabular}

En la etapa de pilotaje se trabajó con la plataforma de manera opcional, los resultados de usuarios que accedieron a la plataforma en esta etapa fueron:

Tabla 3: Resultados etapa de pilotaje

\begin{tabular}{|l|c|c|c|}
\hline & \multicolumn{3}{|c|}{$2016-2017$} \\
\hline AREAS DEL CONOCIMIENTO & AU & DC & ES \\
\hline Ciencias de la Vida & 255 & 255 & 1363 \\
\hline Ciencias Exactas & 308 & 287 & 4132 \\
\hline Ciencias Sociales & 255 & 255 & 2000 \\
\hline \multicolumn{1}{|c|}{ TOTALES } & 818 & 797 & 7495 \\
\hline
\end{tabular}

\section{REFERENCIAS}

AU: Número de aulas virtuales

DC: Número de docentes

ES: Número de estudiantes

La participación en la etapa de pilotaje refleja un inicio con diversos actores, de diferentes áreas del conocimiento y con un alcance representativo en relación al universo, cerca del $20 \%$ de estudiantes son parte de la etapa, y un tercio de docentes al menos a implementado una aula virtual.

Resultado de la implementación total

La comunidad universitaria potencial a ser atendida en esta etapa es de 4.400 estudiantes de nivelación,
37.000 estudiantes de grado, 2.500 estudiantes de posgrado, 2.300 docentes.

Con los roles definidos se realizó un ajuste técnico se volvió a realizar socializaciones en todas las facultades y a cada uno de los administradores por facultad y se habilitaron los siguientes recursos: 1) Curso online masivos y abiertos (MOOC) para uso de la plataforma virtual, disponible para docentes y estudiantes; 2) Módulo para solicitud de creación de curso, los cuales son verificados con el Sistema Académico Universitario para crear correctamente el nombre, asignar los estudiantes y docentes; 3) Creaciones de estructuras por dominios de trabajo es decir grado, posgrado, formación docente.

Los resultados en la etapa de implementación fueron:

Tabla 4: Resultados etapa de implementación total y su incremento respecto de la etapa de pilotaje

\begin{tabular}{|l|c|c|c|c|c|c|}
\hline & \multicolumn{3}{|c|}{ Implementación 2017- } & \multicolumn{3}{|c|}{$\begin{array}{c}\text { Porcentaje de creci- } \\
\text { miento }\end{array}$} \\
\hline $\begin{array}{c}\text { AREAS DEL } \\
\text { CONOCI- } \\
\text { MIENTO }\end{array}$ & AU & DC & ES & AU & DC & ES \\
\hline $\begin{array}{l}\text { Ciencias de la } \\
\text { Vida }\end{array}$ & 804 & 247 & 1664 & $315 \%$ & $145 \%$ & $122 \%$ \\
\hline $\begin{array}{l}\text { Ciencias } \\
\text { Exactas }\end{array}$ & 590 & 300 & 8742 & $192 \%$ & $105 \%$ & $212 \%$ \\
\hline $\begin{array}{l}\text { Ciencias } \\
\text { Sociales }\end{array}$ & 1695 & 465 & 7408 & $665 \%$ & $274 \%$ & $370 \%$ \\
\hline TOTALES & 3089 & 1012 & 17814 & $378 \%$ & $127 \%$ & $238 \%$ \\
\hline
\end{tabular}

\begin{tabular}{|l|}
\hline REFERENCIAS \\
\hline AU: Número de aulas virtuales \\
\hline DC: Número de docentes \\
\hline ES: Número de estudiantes \\
\hline
\end{tabular}

Es destacable el crecimiento desde la etapa de pilotaje a la de implementación total, a nivel de aulas su incremento está cerca de cuadriplicar, en estudiantes sobrepasa al doble de la etapa inicial, en el rubro de participación docente el incremento es menor. Se observa por tanto que entre los docentes que utilizan la plataforma se consolida como un complemento a su labor, por el sector estudiantil el impacto en importante en cuanto se aproxima a la mitad de ellos.

Un análisis que se refiere al género en la participación estudiantil, refleja armonía con la distribución de género en la matrícula, en al área de ciencias de la vida el número de hombres es de 766 y mujeres 898 (46,03\% y 53,97\% respectivamente), en el área de ciencias exactas participaron 4.634 hombres y 4.108 mujeres (53\% y 47\%), y en el área de ciencias sociales 3.160 hombres y 4.248 mujeres $(42,65 \%$ y $57,35 \%$ ). Si provoca la reflexión que motive el incre- 
mento en el área de ciencias de la vida ya que su participación es menor, el trabajo es llegar al docente de esas áreas, puesto que así se aperturarán nuevas aulas y conlleva a la participación estudiantil.

\section{Resultados por recursos creados}

El uso que se dio a las aulas virtuales es diverso, se han obtenido las actividades realizadas y recursos utilizados, con ello se conoce que elementos van tomando relevancia en el apoyo a la docencia y al aprendizaje estudiantil.

Los resultados de los usuarios, docentes y estudiantes, están referenciados a grado, se han montado los cursos en grado y un curso general para nivelación (Ética y Pensamiento Universitario), a futuro deberá llegar a universalizar en tercer nivel y ser utilizada en cuarto nivel, en éste nivel hoy prácticamente está desconocida.

Los datos referentes al trabajo docente son obtenidos del aplicativo uvirtual, en el caso del período 20162017 incluye a seis meses de actividades, en el período 2017-2017 incluye tres meses en pues el período está en marcha, estos presentan los siguientes resultados sobre las actividades trabajadas en la preparación de recursos:

Tabla 5 Actividades realizadas en Aula Virtual

\begin{tabular}{|l|r|r|r|}
\hline RECURSO & 2016-2017 & 2017-2017 & \% DE USO \\
\hline Archivo & 3714 & 3815 & 16,343 \\
\hline Asistencia & 1300 & 2206 & 7,610 \\
\hline $\begin{array}{l}\text { Base de } \\
\text { datos }\end{array}$ & 18 & 5 & 0,050 \\
\hline Carpeta & 352 & 383 & 1,595 \\
\hline $\begin{array}{l}\text { Cuestion- } \\
\text { ario }\end{array}$ & 1664 & 2239 & 8,472 \\
\hline Curso & 1721 & 4053 & 12,534 \\
\hline Etiqueta & 2471 & 2130 & 9,987 \\
\hline Foro & 1016 & 991 & 4,357 \\
\hline Glosario & 27 & 184 & 0,458 \\
\hline Lección & 60 & 66 & 0,274 \\
\hline Libro & 165 & 75 & 0,521 \\
\hline Taller & 256 & 227 & 1,048 \\
\hline Tarea & 2585 & 2853 & 11,804 \\
\hline URL & 824 & 1327 & 4,669 \\
\hline Wiki & 39 & 34 & 0,158 \\
\hline $\begin{array}{l}\text { Crear mód- } \\
\text { ulo/curso }\end{array}$ & 3526 & 2945 & 14,047 \\
\hline Página & 200 & 917 & 2,425 \\
\hline Chat & 88 & 85 & 0,376 \\
\hline Consulta & 95 & 61 & 0,339 \\
\hline
\end{tabular}

\begin{tabular}{|l|r|r|r|}
\hline Categoria & 394 & 853 & 2,707 \\
\hline Skype & 6 & 1 & 0,015 \\
\hline Botones & 33 & 17 & 0,109 \\
\hline Encuesta & 12 & 11 & 0,050 \\
\hline $\begin{array}{l}\text { Herramien- } \\
\text { ta externa }\end{array}$ & 0 & 1 & 0,002 \\
\hline Quiz offline & 0 & 1 & 0,002 \\
\hline Insignia & 12 & 10 & 0,048 \\
\hline TOTALES & $\mathbf{2 0 5 7 8}$ & $\mathbf{2 5 4 9 0}$ & $\mathbf{1 0 0}$ \\
\hline
\end{tabular}

Las principales actividades que se realizan se asocian al diseño mismo del curso, predominan el subir archivos, crear los cursos o módulos, organizar cursos, crear etiquetas, registrar asistencias, registros de URL; entre las anteriores se contiene el 65,19 por ciento de actividades. Las actividades que orientan a una mayor actividad de acción del estudiante como: tareas, cuestionarios, foros (ocupan la cuarta, sexta o novena posición) con un 24,63 por ciento. Otras actividades son de menor rango.

Para el análisis de trabajo por parte de los estudiantes, se obtuvo la información de dos meses (octubre y noviembre de 2016), sus resultados se resumen así:

Tabla 6 Recursos utilizados por los estudiantes

\begin{tabular}{|l|r|r|}
\hline RECURSO UTILIZADO & TOTAL GENERAL & PORCENTAJE \\
\hline Archivo & 21074 & 9,341 \\
\hline Asistencia & 327 & 0,145 \\
\hline Base de datos & 9 & 0,004 \\
\hline Carpeta & 1413 & 0,626 \\
\hline Chat & 1113 & 0,493 \\
\hline Cuestionario & 22796 & 10,104 \\
\hline Foro & 158989 & 70,469 \\
\hline Glosario & 6637 & 2,942 \\
\hline Lección & 22 & 0,010 \\
\hline Libro & 1592 & 0,706 \\
\hline Pagina & 790 & 0,350 \\
\hline Taller & 163 & 0,072 \\
\hline Tarea & 4015 & 1,780 \\
\hline Texto en línea & 956 & 0,424 \\
\hline URL & 4016 & 1,780 \\
\hline Video conferencia & 126 & 0,056 \\
\hline Wiki & 1576 & 0,699 \\
\hline & $\mathbf{2 2 5 6 1 4}$ & $\mathbf{1 0 0}$ \\
\hline TOTALES & &
\end{tabular}

De lejos el recurso con más utilización es el foro $(70,47 \%)$, seguidos del cuestionario $(10,10 \%)$ y el manejo de archivos $(9,34 \%)$; los otros doce recursos solo representan el $10 \%$ restante. 
El soporte continuo, tanto a nivel técnico como administrativo es proporcionado para los estudiantes y profesores. Para ello la DTIC tiene preparado al personal técnico.

\section{Conclusiones}

Para promover el uso de una plataforma virtual en la Universidad Central se han tomado acciones y se han conseguido algunos aprendizajes significativos, entre ellos se señalan los siguientes:

1. La implementación de la plataforma no tuvo inconvenientes técnicos, pues se contó con una infraestructura de tecnológica sólida, la confianza en el uso de la misma en la comunidad universitaria ha permitido el crecimiento de usuarios. El acceso de los usuarios es 24/7, con ello se permite acceder a estudiantes y docentes a sus cursos según sus necesidades, requerimientos y ritmos.

2. Se determinó a la plataforma Moodle como la que presta mejor servicio, se apoya la decisión por contar con código abierto, muy buena usabilidad, popularidad, soporte, adaptabilidad y capitalización.

3. Las herramientas más utilizadas por parte de los estudiantes son: el foro, el cuestionario, el archivo. Por el lado de los docentes el archivo, creación de cursos, organización de cursos y tareas. En el estudio del uso de la plataforma deberá investigarse como se los utiliza y proponer procedimientos de mejora.

4. Es necesario realizar estudios del cómo son utilizados para orientar en la mejor explotación de la plataforma.

5. Es necesario diseñar un Plan de Capacitación Continua para el docente con el propósito de establecer acciones de mejora continua. El docente de la UCE debe convertirse en actor del uso de la plataforma.

De la aplicación hay varias recomendaciones a futuro, algunas se han señalado en las conclusiones, pero se añade una que está latente y se refiere a estudiar el impacto en los docentes, el impacto en los estudiantes; en ambos casos deberá acompañarse de los problemas que tuvieron frente al uso de la plataforma y la facilidades que encontraron; también habrá que estudiar su impacto en los resultados académicos en el aprendizaje.

\section{Referencias bibliográficas}

Ardila Rodríguez, M. (2012). Son las Plataformas Digitales un componente determinante en la calidad de la formación en ambientes virtuales. Educación y Desarrollo Social, 10.

Cabero, J. (2008). Tecnología Educativa. Barcelona: Síntesis.

Chanona Burguete, O. (2017). Digitalidad: cambios y mutaciones en la cotidianidad. Revista Digital Universitaria, 11.

Instituto Internacional de la UNESCO para la Educación Superior en América Latina. ( para la Educación Superior en América Latina). La Educación Superior Virtual en América Latina y el Caribe. Caracas: ESALC/UNESCO.

Jódar Marín, J. Á. (2010). La era digital: Nuevos Medios, Nuevos Usuarios, Nuevos Profesionales. Razón y Palabra, 17.

Latorre, A. (2003). La investigación-acción. Conocer y cambiar la práctica educativa.

Loaiza, R., \& Arévalo, M. (2010). Metodología para la implementación de Proyectos E-Learning. Universidad de Carabobo, 12

Montero López, M., \& Licona Alcalan, K. (2016). El impacto del proceso de acreditación del programa de la Licenciatura en Educación Física de la Normal Superior en Tapachula. Debates en Evaluación y Curriculum, 10.

Pérez Cervantes, M., \& Saker Barros, A. (2012). análisis de la efectividad del uso de la. Red de Revistas Científicas de América Latina, el Caribe, España y Portugal, 18.

Secretaría Nacional de Ciencia y Tecnología. (01 de 01 de 2017). Consejo de Evaluación, Acreditación y Aseguramiento de la Calidad de la Educación Superior. Obtenido de http:// www.ceaaces.gob.ec/sitio/wp-content/uploads/2013/10/ rloes1.pdf

Sistema de Información de Tendencias Educativas en América Latina. (2015). Revolución digital. Ciudadanía y derechos en construcción. Sistema de Información de Tendencias Educativas en América Latina, 25. 